\title{
The surgical management of congenital kyphosis and kyphoscoliosis
}

\begin{abstract}
Study Design: A retrospective study of surgery for congenital kyphosis and kyphoscoliosis.

Objective: To assess the effectiveness of different types of spine surgery in the management of congenital kyphosis and kyphoscoliosis.

Summary of Background Data: Congenital kyphosis and kyphoscoliosis are much less common than congenital scoliosis but potentially more serious, because these curves can progress rapidly and Type I deformities can lead to spinal cord compression and paraplegia. No one operative procedure can be applied to all types and sizes of deformity. The method of surgical treatment depends on the age of the patient, the type and size of the deformity, and the presence or absence of spinal cord compression causing a neurologic deficit.

Methods: Sixty-five patients with a congenital kyphosis $(n=14)$ or kyphoscoliosis $(n=51)$ were treated by five different methods of spine arthrodesis: prophylactic posterior arthrodesis before age of 5 years $(n=11)$, posterior arthrodesis after age 5 years without instrumentation $(\mathrm{n}=26)$ and with instrumentation $(\mathrm{n}=12)$, combined anterior and posterior arthrodesis without instrumentation $(n=7)$ and with instrumentation $(n=9)$. Six patients had preoperative lower limb spastic paraparesis caused by spinal cord compression. The mean age at surgery was 9 years 6 months (range, 11 months to 25 years), and all 65 patients were observed for a minimum of 2 years (mean 6 years 6 months, range 2 to 18 years). Fifty-seven patients reached skeletal maturity.
\end{abstract}

Results: A posterior arthrodesis performed before the age of 5 years resulted in a gradual reduction of the kyphosis by a mean $15^{\circ}$ in 9 of the 11 patients, followed up for a mean of 11 years, whose initial kyphosis was less than $55^{\circ}$. Patients treated after the age of 5 years by a posterior arthrodesis followed by cast application had poor correction and a high incidence of pseudarthrosis. This was not significantly improved by the addition of posterior instrumentation. For curves greater than $60^{\circ}$, the most successful results were achieved by an anterior spinal release and arthrodesis with strut graft correction followed by posterior arthrodesis with instrumentation (if possible).

Conclusion: All patients with a Type I or Type III congenital kyphosis or kyphoscoliosis should be treated by a posterior arthrodesis before the age of 5 years and before the kyphosis exceeds $50^{\circ}$. A kyphosis that does not reduce to less than $50^{\circ}$ as measured on the lateral spine radiograph made with the patient supine requires an anterior release and arthrodesis with strut grafting followed by posterior arthrodesis with instrumentation (if possible).

Keyword: Congenital kyphosis; Surgery 